\title{
Análise de estruturas de suporte para sinalizações viárias
}

\section{Support structures analysis for highway signs}

DOI: $10.46814 /$ lajdv3n5-048

Recebimento dos originais: 01/09/2021

Aceitação para publicação: 01/10/2021

\section{César Alberto Chagoyen Méndez}

Doutor

Facultad de Ingeniería Mecánica, Universidad Central "Marta Abreu" de Las Villas.

Carretera a Camajuaní, km 51⁄2, Santa Clara, Villa Clara, Cuba, 54830.

E-mail: cachagoyen@uclv.edu.cu

\section{Sergio Alejandro Rojas Pérez}

Engenheiro Mecânico

Facultad de Ingeniería Mecánica, Universidad Central "Marta Abreu” de Las Villas.

Carretera a Camajuaní, km 51⁄2, Santa Clara, Villa Clara, Cuba, 54830.

E-mail: srperez@uclv.cu

\section{Ernesto Luciano Chagoyen Méndez}

Doutor

Facultad de Construcciones, Universidad Central "Marta Abreu" de Las Villas.

Carretera a Camajuaní, km 51⁄2, Santa Clara, Villa Clara, Cuba, 54830.

E-mail: chagoyen@uclv.edu.cu

\section{Constantina Álvarez Peña}

Doutor

Departamento de Ingeniería Eléctrica. Universidad de Oviedo, Campus Viesques.

Bloque 3.1.10 - (33204), Gijón - Asturias, España.

E-mail: tina@uniovi.es

\section{Aristides Rivera Torres}

Doutor

Departamento de Engenharia Mecânica, Universidade do Estado do Amazonas, Manaus, AM

Ave. Darcy Vargas 1200, Parque 10, Manaus AM, CEP 69050-020

E-mail: artorres@uea.edu.br

\section{Antônio Claudio Kieling}

Doutor

Departamento de Engenharia Mecânica, Universidade do Estado do Amazonas, Manaus, AM

Ave. Darcy Vargas 1200, Parque 10, Manaus AM, CEP 69050-020

E-mail: akieling@uea.edu.br

\section{Marcos Dantas do Santos}

Mestre

Departamento de Engenharia Mecânica, Universidade do Estado do Amazonas, Manaus, AM

Ave. Darcy Vargas 1200, Parque 10, Manaus AM, CEP 69050-020

E-mail: mdsantos@uea.edu.br 


\title{
Gabriel Federico Rivero Llerena
}

Doutor

Departamento de Engenharia Mecânica, Universidade do Estado do Amazonas, Manaus, AM

Ave. Darcy Vargas 1200, Parque 10, Manaus AM, CEP 69050-020

E-mail: gllerena@uea.edu.br

\section{RESUMO}

El cálculo de las estructuras de soporte para señalizaciones viales se realiza mediante la norma AASHTO con la cual se realiza fundamentalmente el chequeo a Fatiga, pero con ella no se llegan a conocer las tensiones von Mises, las tensiones tangenciales ni los desplazamientos en cualquier punto de la estructura. En el presente trabajo se determinan las cargas actuantes, así como sus combinaciones más críticas para el análisis. Se realiza el cálculo analítico a través de los métodos y las normas establecidas para ello y se confecciona un modelo de elementos finitos cuya simulación numérica complementa los resultados obtenidos por las normas.

Palavras-chave: estructuras de soporte, señalizaciones viales, simulación numérica, elementos finitos.

\begin{abstract}
The calculation of the support structures for highway signs is carried out by means of the AASHTO standard, with which the Fatigue check is fundamentally carried out, but with it the von Mises stress, the tangential stress and the displacements at any point of the structure are not known. In the present work, the acting loads are determined, as well as their most critical combinations for the analysis. The analytical calculation is performed through the methods and standards established for it and a finite element model is created whose numerical simulation complements the results obtained by the standards.
\end{abstract}

Keywords: structural supports, highway signs, numeric simulation, finite elements.

\section{INTRODUÇÃO}

Las primeras señales de tráfico datan de la época de los romanos, que fueron los pioneros al establecer un código de señales para orientar al viajero en el tránsito por las calzadas. Estas señales de tráfico romanas se llaman millarios y contaban en millas romanas la distancia desde ese punto a los diferentes destinos que comunicaba la vía o calzada. Los millarios eran habitualmente grandes columnas de piedra de una pieza en las que se labraba a cincel y en números romanos, por supuesto, los destinos, las distancias, el nombre de la vía y en muchos casos el financiador de tal calzada.

Este código de orientación se complementaba con el primer mapa de carreteras conocido. Durante toda la edad media y hasta casi el siglo XX las señales de tráfico de los caminos apenas variaron de los códigos usados por los romanos. Se seguían utilizando mojones de piedra grabados con la información, en todo caso se añadieron cartelones en otros materiales como el hierro o la madera, pero se avanzó poco en este sentido. [1] 
Los patrones básicos de las señales de tráfico se establecieron en el 1908 en el Congreso Internacional de Carreteras, en Roma. Ya en 1968, los países europeos firmaron en la Convención de Viena un Tratado sobre circulación por carretera, con el objetivo de estandarizar las normas de tráfico en los países participantes, facilitar el tráfico internacional por carretera y aumentar la seguridad vial. En la actualidad el Tratado cuenta con 52 países signatarios, en los que se incluye toda Europa, gran parte de Asia y muchos países africanos. [1]

Estados Unidos desarrolló su propio sistema de señalización vial, que también fue adoptado por otras naciones (con variaciones propias de cada país, este sistema está implementado en toda América y casi la totalidad de Oceanía y algunos países africanos). Los primeros intentos de normalización de las señales de tráfico norteamericanas se producen en 1905. El Automóvil Club Búfalo instaló una extensa red de señales de tráfico en el estado de Nueva York. En 1909, el Club de Automóviles de California emprendió la tarea de señalizar las principales carreteras en un radio de 250 millas en torno a San Francisco. A principios de la década de 1920, los representantes de Wisconsin, Minnesota e Indiana recorrieron varios Estados con la intención de desarrollar una base uniforme de signos y marcas viales. En 1935, se publicó la primera guía de normas de tráfico a nivel interestatal la cual ha crecido y revisado aproximadamente cada diez años. Con el incremento de los medios de transporte las vías se hicieron mayores y más peligrosas y con estas las señalizaciones de tránsito aumentaron en cuanto a tamaño y cantidad, por lo que el estudio de sus estructuras de soporte comienza a jugar un papel importante para el trabajo ingenieril. [1]

Las estructuras de soporte para las señalizaciones viales son diseñadas con la finalidad de soportar y dar rigidez a los tableros y demás elementos que pudieran tener dichas señales, dependen de su tipo, tamaño ubicación y características del terreno, así como de las presiones y succiones debidas al viento a que están sujetas, por lo que se debe elaborar un proyecto particular en cada caso en relación a su cimentación y estructura de soporte teniendo en cuenta la norma de diseño ASCE/SEI 48-2019, las especificaciones para las estructuras de soporte y sus revisiones [2] y la guía de instalación FHWA NHI 05-036 [3]. Las mismas se clasifican en Postes y Estructuras, de estas últimas aparecen las rigidizantes y las de soporte tipo voladizo, tipo bandera, tipo bandera doble y tipo puente. Estos junto a los tableros, elementos de sujeción, tornillos y anclas deben ser de acero galvanizado [3].

Para el cálculo de las estructuras de soporte se emplea la norma AASHTO (American Association of State Highway and Transportation Officials) denominada "Standard Specifications for Structural Supports for Highway Signs, Luminaries and Traffic Signals” [2], la cual es actualizada anualmente. Con esta norma se realiza fundamentalmente el chequeo a Fatiga, pero no se llega a determinar las tensiones von Mises, las tensiones tangenciales ni los desplazamientos en cualquier punto de la estructura, que se realizan en este trabajo. 
Para determinar el comportamiento completo de la estructura de soporte para la señalización vial, que es lo que se presenta en este trabajo, fue necesario inicialmente determinar las cargas que actúan sobre la estructura (viento natural y ráfagas producidas por los camiones) y las combinaciones más críticas para su análisis. Posteriormente, se realizó el cálculo analítico a través de las normas AASHTO establecidas para ello, elaborando un diagrama de flujo de los cálculos y confeccionando una hoja de cálculo.

Se realizó un modelo de elementos finitos utilizando el software SolidWorks para simular numéricamente la estructura de soporte bajo las cargas determinadas. El Método de los Elementos Finitos (MEF) es ampliamente utilizado para simular estructuras, el contacto entre diferentes materiales, etc. y para predecir diversos parámetros importantes en diferentes ramas de la ciencia y la ingeniería como la biomecánica [4], tratamientos térmicos [5-6], materiales [7], materiales compuestos [8-9], etc. Como resultado del trabajo se obtuvo el comportamiento completo de la estructura, las curvas de variación de los desplazamientos y las tensiones, así como las ecuaciones que describen su comportamiento.

\section{MATERIAIS E MÉTODOS}

\subsection{TIPOS Y EFECTOS PRINCIPALES DE LAS CARGAS}

Las cargas de diseño para estas estructuras incluyen: cargas muertas y vivas, cargas de hielo, y cargas de viento, siendo estas últimas las que mayor influencia tienen en el diseño. Las cargas muertas incluyen el peso propio del soporte estructural, así como también el peso de las señales, las luminarias y semáforos, carteles informativos y cualquier otro dispositivo o accesorios permanentemente conectado a la estructura. Las cargas temporales que pueden ocurrir durante el mantenimiento también deben ser consideradas como cargas muertas. Las cargas de hielo se tienen en cuenta considerando un espesor de hielo equivalente a 15,24 $\mathrm{mm}$ aplicado a todas las superficies, pues las estructuras localizadas en zonas donde nieva se consideran vulnerables a esta carga. [10]

Los tres fenómenos más importantes producidos por las cargas del viento que pueden conducir a la vibración y la fatiga son: las ráfagas naturales, las ráfagas inducidas por camiones y el galopeo. La interacción de una estructura de soporte con el viento depende de su rigidez y su forma. Por ejemplo, el galopeo puede ocurrir en estructuras voladizas y no en estructuras con forma de puente.

Ráfagas naturales de viento: aportan las vibraciones más comúnmente inducidas en las estructuras de soporte. Ejercen una fuerza fluctuante que es principalmente horizontal, por lo que el movimiento resultante del Brazo también lo es, aunque puede aparecer un movimiento vertical igualmente significativo. La presión de las ráfagas naturales es aplicada horizontalmente en el área 
frontal proyectada por todas las superficies, incluyendo los miembros estructurales y las señales. El agrietamiento por fatiga es la falla que se produce y se desarrolla en un período de tiempo relativamente corto. En los voladizos las grietas usualmente se manifiestan en la conexión del Brazo con el Poste con grietas que se forman a lo largo de la conexión. En los lugares donde la velocidad del viento alcanza valores máximos, pero no constantes, la posibilidad de falla por fatiga es mínima.

Ráfagas inducidas por camiones: el paso de camiones bajo las estructuras de soporte induce cargas horizontales y verticales, estas no crean un movimiento primordialmente vertical, sino que también pueden incluir un componente horizontal igualmente significativo. Debido al gran tamaño de los carteles y señales, las estructuras de soporte son más susceptibles para la fatiga inducida por ráfagas de camiones ya que le donan una gran área en el plano horizontal. Las grietas de la fatiga causada se desarrollan en la conexión del Brazo con el Poste y en la base del Poste, esto ocurre en un período de varios años. [11]

Galopeo: Las acciones del galopeo provocan la aparición de oscilaciones en dirección perpendicular al flujo del viento. A diferencia de otros efectos este ocurre en miembros asimétricos (señales, letreros y otros anexos). Por consiguiente, el Brazo es más susceptible al galopeo que el Poste. En ocasiones el galopeo ha causado que Brazos de ciertas estructuras se muevan de arriba abajo con un rango mayor que 1 metro. Para que este fenómeno ocurra se requieren vientos estables y uniformes en vez de ráfagas fuertes e inestables. Las cargas galopantes son realmente severas, por lo que cuando se tienen en cuenta, prácticamente gobernarán el diseño por fatiga. Por consiguiente, los dispositivos de amortiguación tienen un costo-beneficio significativo al reducir los efectos del galopeo, contrario a sus efectos ante las ráfagas de viento natural o de camiones. En la mayoría de los casos que han sido investigados, las grietas de fatiga provocadas por galopeo se desarrollan durante una larga temporada de un año o más, donde ocurrieron varios días de vientos hostigadores [12].

Como se ha podido apreciar en la descripción de los tipos y efectos principales de las cargas todos apuntan a la fatiga como falla principal. Es por ello que, cuando se emplean las normas AASHTO se realizan los chequeos de fatiga principalmente en la base del Brazo y del Poste bajo estos tipos de cargas.

\subsection{CÁLCULO ANALÍTICO DE ESTRUCTURAS MEDIANTE LAS NORMAS AASHTO}

Una descripción simplificada del cálculo de estructuras elevadas de soporte de señalización vial mediante las normas AASHTO se puede apreciar en el Diagrama de Flujo que aparece representado en la figura 1, el cual constituyó una guía para el trabajo posterior. 
El cálculo comienza con las Definiciones y Suposiciones tales como las características del material de la estructura, los datos geométricos y el peso propio, entre otros. Luego se definen las Cargas y las suposiciones consideradas. Pueden ser:

Cargas Muertas: para las cuales hay que definir el peso y las acciones tanto en el Brazo como en el Poste, considerando básicamente las Señales (semáforos) y los Letreros.

Cargas de Viento: estado límite: Extremo I.

Cargas Factoradas y Chequeo de Resistencia tanto en Brazo como en el Poste. Posteriormente a estos dos cálculos se realiza el cálculo de la Soldadura en la Base del Brazo y el Diseño de la Chapa Base del Poste.

A continuación, se calculan los Momentos Totales para el Cálculo a Fatiga en cada uno de los ejes correspondientes (tanto en la Base del Brazo como en la Base del Poste) debido a Fatiga por ráfagas naturales de viento, Fatiga por ráfagas inducidas por camiones y Fatiga por galopeo.

Ya con los momentos Totales, se puede realizar el Chequeo a Fatiga de la Base del Brazo y de la Base del Poste.

Fig. 1 Diagrama de Flujo para el cálculo analítico (normas AASHTO).

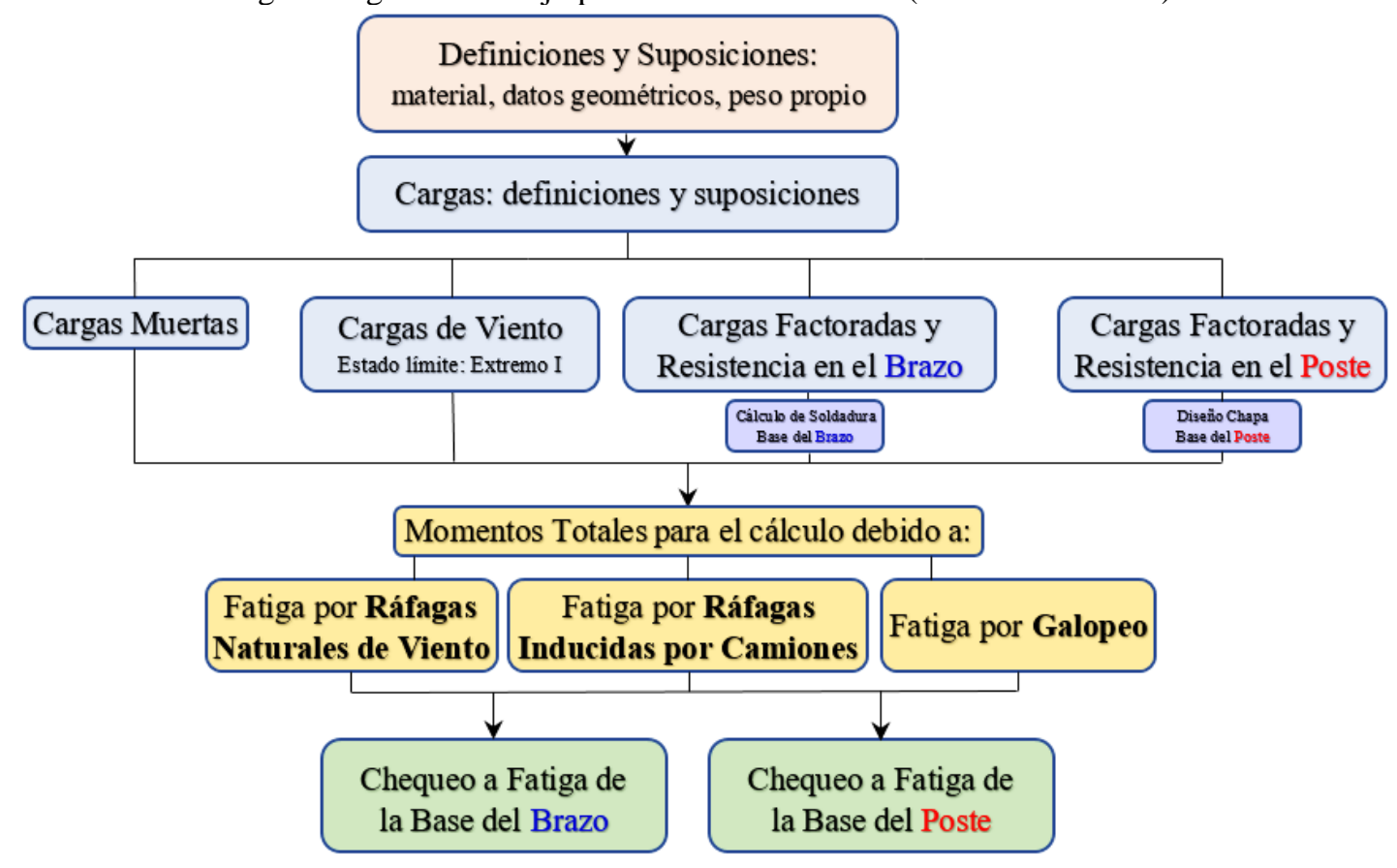

A partir del Diagrama de Flujo descrito anteriormente se elaboraron 15 hojas de cálculo en Excel enlazadas que facilitaron el procesamiento de toda esta información y los cálculos en forma matricial, debido a la existencia de varias señales y semáforos, de tamaño y dimensiones diferentes (170 datos y 450 expresiones). Un ejemplo de estas hojas de cálculo es la que se muestra en la figura 2 con el Chequeo a Fatiga en la Base del Brazo, respectivamente. 
Fig. 2 Imagen de la hoja Excel para el Chequeo a Fatiga en la Base del Brazo.

\begin{tabular}{|c|c|c|c|c|c|c|}
\hline \multicolumn{7}{|c|}{ Cálculo Analítico (norma AASHTO) de Estructuras elevadas de Soporte de Señalización Vial } \\
\hline \multicolumn{3}{|c|}{ Conección de la Base del Brazo, Detalles y Definiciones } & \multicolumn{4}{|c|}{ Chequeo de Fatiga } \\
\hline$A_{\text {botts }}($ in $)=$ & 12 & & \multicolumn{2}{|c|}{ Rango de tensión en la soldadura de la placa con la base del brazo } & \multicolumn{2}{|c|}{ Rango de tensión en los pernos de la conección de la placa con la base del brazo } \\
\hline$B_{\text {bots }}(\mathrm{in})=$ & 20 & & $\sigma_{\text {FAT_Gal_weld }}(\mathrm{ksi})=$ & 7.2 & $A_{\text {bott }}$ (in) $=$ & 12 \\
\hline$\Delta \mathrm{F}_{\mathrm{TH} \_ \text {arm }}(\mathrm{ksi})=$ & 7 & & GallopingRatio= & 0.72 & $B_{\text {boit }}($ in $)=$ & 20 \\
\hline$t_{a r m \_p l a t e}(i n)=$ & 2 & & ArmWeldCheck $_{\mathrm{Gal}}=$ & & area $_{\text {bolt }}\left(\mathrm{in}^{2}\right)=$ & 1.41 \\
\hline$A_{\text {arm_fatigue }}\left(\mathrm{ksi}^{3}\right)=$ & 2200000000 & & & & $n_{\text {bott }}($ in $)=$ & 4 \\
\hline $\mathrm{D}_{\mathrm{T}}(\mathrm{in})=$ & 12.31 & & $\sigma_{\text {FAT_NW_weld }}(\mathrm{ksi})=$ & 2.86 & $\mathrm{I}_{\text {long }}\left(\mathrm{in}^{4}\right)=$ & 564 \\
\hline$D_{B C}($ in $)=$ & 24 & & NaturalGustRatio $=$ & 0.29 & $I_{\text {short }}\left(\right.$ in $\left.^{4}\right)=$ & 203.04 \\
\hline $\mathrm{N}_{\mathrm{B}}=$ & 4 & & \multirow[t]{2}{*}{ ArmWeldCheck $_{\text {NWW }}=$} & & $\sigma_{\text {FAT_Ga!_botst }}(\mathrm{ksi})=$ & 2.71 \\
\hline$t_{T}($ in $)=$ & 0.31 & & & & GallopingBoltRatio= & 0.39 \\
\hline$t_{T P}(i n)=$ & 2 & & $\sigma_{\text {FAT_TG_weid }}(\mathrm{ksi})=$ & 0.82 & ArmBoltCheck $_{\mathrm{Ga}}=$ & \\
\hline $\mathrm{D}_{\text {Op }}(\mathrm{in})=$ & 3 & & TruckGustRatio= & 0.08 & & \\
\hline$C_{O P}=$ & 0.24 & & ArmWeldCheck $_{\text {TG }}=$ & ok & $\sigma_{\text {FAT NW bots }}(\mathrm{ksi})=$ & 4.23 \\
\hline 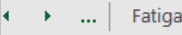 & Rafagas-Natur & & iga Rfgas-Natur Base-Poste $\quad$ Fatiga & imion & Fatiga Rfgas-Camion Base-Poste & Fatiga-Base del Brazo \\
\hline
\end{tabular}

Para validar estas hojas de cálculo se evaluó una estructura de soporte de señalizaciones de transito localizada en Chicago, Illinois, que permite la comprobación de las expresiones introducidas en las hojas. Esta estructura se encuentra resuelta analíticamente y publicada en el Apéndice C del Reporte NCHRP Report 796 [13] y en NCHRP Report 494 [14]. En NCHR Report 469 [15] aparecen otros ejemplos de estructuras. Este análisis se basa en las normas AASHTO.

La estructura es del tipo voladizo y soporta tres semáforos y cuatro señales (anexos). Las dimensiones geométricas de la estructura, así como la posición de los semáforos y señales se puede apreciar en la figura 3. La estructura está localizada en una autopista donde el límite de velocidad es de $72.42 \mathrm{~km} / \mathrm{h}$ (45 mph) y ocurren vientos frecuentes por lo que el estado de fatiga límite debe ser comprobado.

Fig. 3 Dimensiones geométricas de la estructura a calcular y ubicación de los anexos. Fuente: [13]

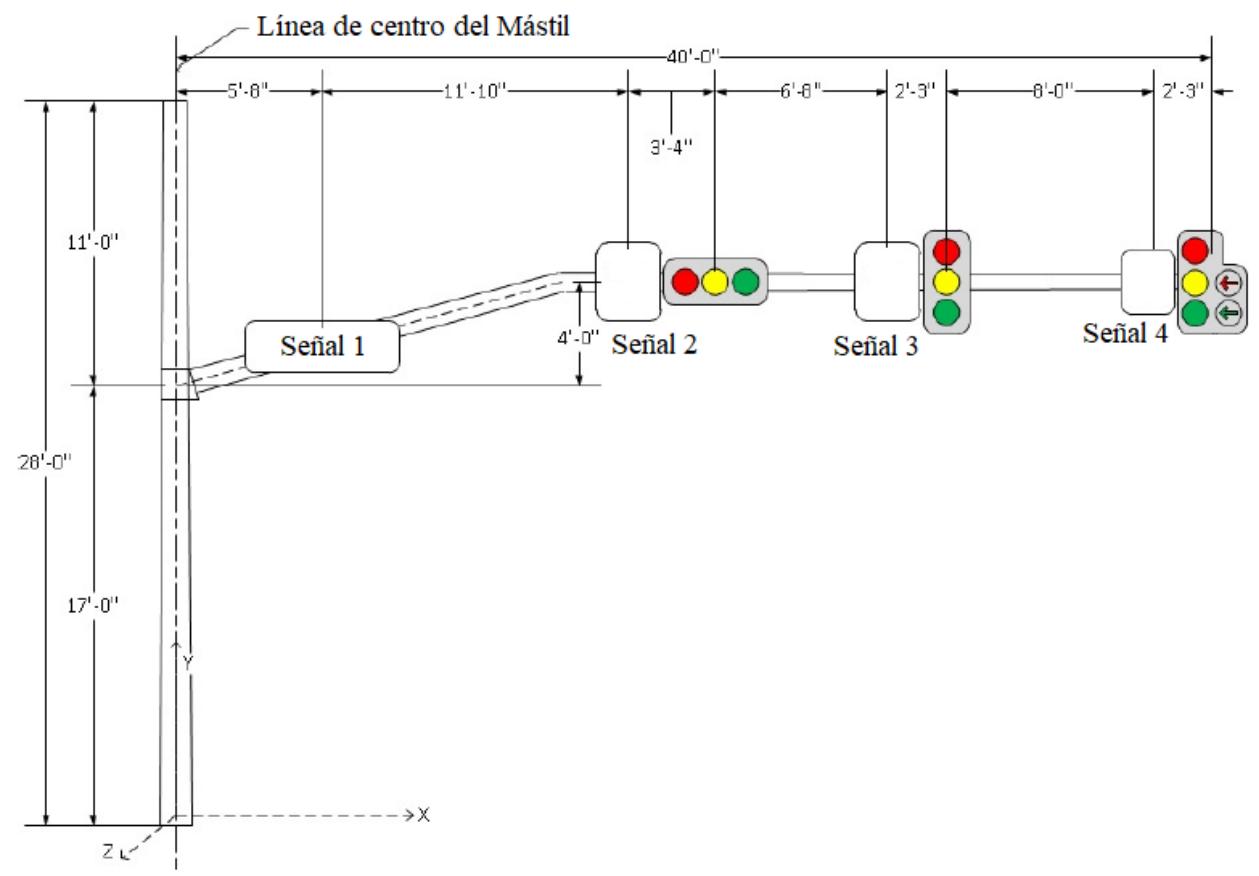


Finalmente se comprobaron las tensiones provocadas por galopeo y ráfagas de viento (naturales e inducidas por camiones) en los tornillos de la unión Brazo-Poste y en los de la base del Poste, con resultados satisfactorios. Los chequeos de las tensiones de fatiga en las soldaduras de la Base del Poste y la Base del Brazo también arrojaron buenos resultados y muy similares a los del ejemplo del Reporte mencionado.

Una conclusión interesante de este cálculo es que las tensiones provocadas por galopeo tanto en las soldaduras como en los tornillos, aunque no llegan a fallar, alcanzan un valor mayor que las originadas por ráfagas de viento. Como se mencionó anteriormente con esta norma se realizan chequeos de fatiga en distintos puntos de la estructura, pero hay parámetros que no se llegan a conocer a través de ella, como son: los desplazamientos que ocurren en la estructura, las tensiones von Mises y las tensiones tangenciales que surgen, así como las deformaciones, entre otros.

Teniendo en cuenta los criterios, conceptos e insuficiencias detectadas en el procedimiento seguido y descrito anteriormente, se elabora un modelo de elementos finitos para poder obtener a partir de la simulación numérica, un conjunto de resultados tan importantes como los mencionados anteriormente [16].

Se emplea el software profesional SolidWorks, el cual es ampliamente utilizado internacionalmente en este tipo de tareas [17]. En la bibliografía revisada aparece el software "SABRE" (Sign bridge analysis and evaluation systems) de Bridge Engineering Software \& Technology Center de la University of Maryland, USA, que permite realizar estos y otros cálculos, pero por su precio se encuentra fuera de nuestro alcance.

\subsection{MODELADO Y SIMULACIÓN DE LA ESTRUCTURA DE SOPORTE}

\subsubsection{Invariante: Geometría}

Como se mencionó anteriormente la estructura soporta tres semáforos y cuatro señales o avisos de tráfico de las cuales se conoce su posición y peso. Está formada por dos elementos principales: el Poste y el Brazo. El primero tiene una altura de 8,5344 m (28 ft) mientras que el segundo posee 12,19 $\mathrm{m}$ (40 ft) de longitud.

En el lugar donde se encuentra la estructura pueden existir vientos que alcanzan $185 \mathrm{~km} / \mathrm{h}(115$ mph), los cuales pueden representar un huracán de categoría 3 de acuerdo a la escala de Saffir/Simpson.

El Poste posee una forma cónica con un diámetro en la base de $381 \mathrm{~mm}$, este tiene un factor de reducción de 11,66 $\mathrm{mm} / \mathrm{m}$ por lo que su diámetro en la punta es de 281,43 $\mathrm{mm}$. El Brazo al igual que el Poste es cónico con el mismo factor de reducción, su diámetro en la base es de 304,8 mm y en la punta $164,08 \mathrm{~mm}$, este tiene en su comienzo una ligera inclinación de 15 grados. La unión entre el Brazo y el Poste se encuentra a 5,186 m de la Base del Poste. Esta unión posee una chapa de 381x605,79 
mm con un espesor de 50,8 mm. Con dichos datos se construyó el modelo geométrico de la estructura el cual aparece representado en la figura 4. En dicha figura aparece un detalle de la unión Brazo-Poste.

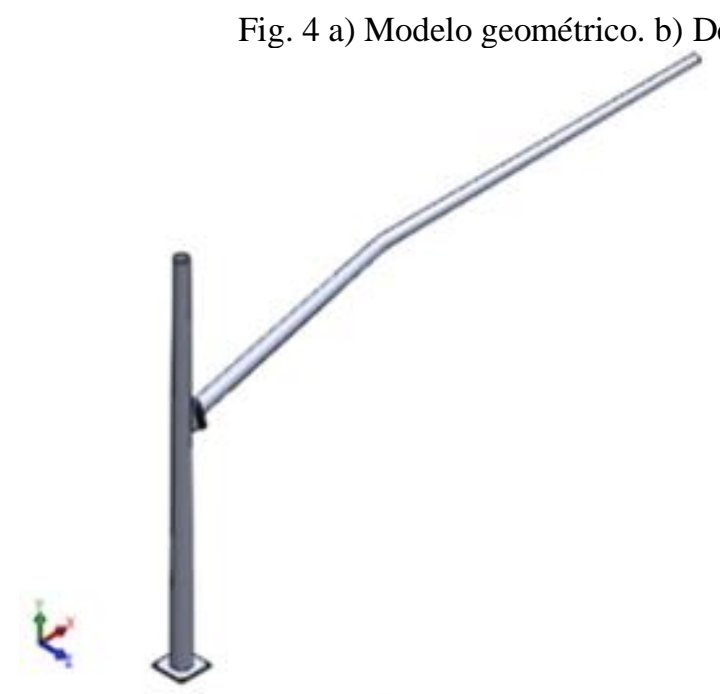

a)

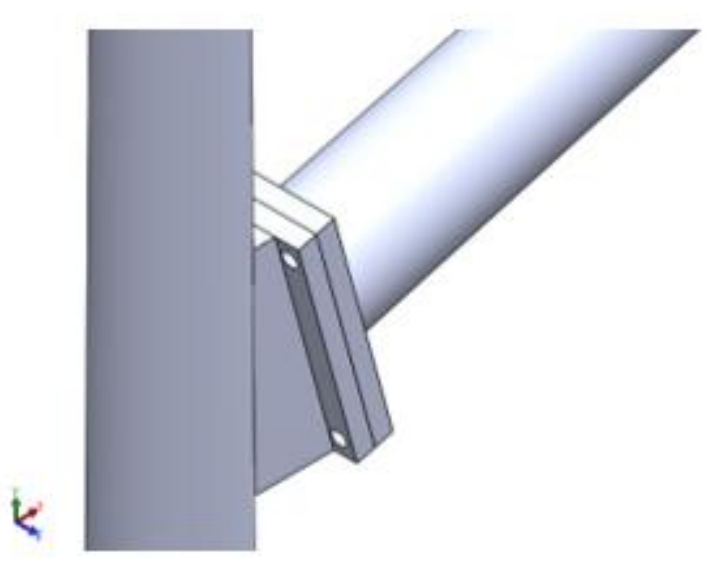

b)

\subsubsection{Invariantes: Restricciones y Cargas}

Las restricciones impuestas al modelo son del tipo "Geometría Fija" y fueron colocadas en el interior de los cuatro agujeros de la placa base, donde van los tornillos de fijación. En la figura 5 se pueden observar las restricciones y un detalle de las mismas.

Esta estructura será simulada con los mismos tipos de carga que fue calculado el ejemplo del Reporte, es decir, cargas muertas y cargas de viento. Las cargas muertas son provocadas por el peso propio de los elementos que intervienen en la estructura ya sea la estructura propiamente dicha o los elementos asociados a ella, que en este caso son tres semáforos y cuatro letreros. Estas cargas son normales al plano que contiene el apoyo de la estructura. Las cargas de viento son las provocadas como su nombre lo dice por la incidencia del viento sobre el área de los elementos de la estructura y sobre el área de la estructura propiamente dicha. Estas fuerzas tienen la misma dirección del viento [18], [19].

En la figura 5 se observa un esquema de las cargas que actúan en el modelo para una velocidad del viento de $185 \mathrm{~km} / \mathrm{h}$. Las cargas señaladas en color lila son las cargas de viento sobre los semáforos y los letreros (2006; 1179; 1468; 1179; 1468; 787 y 2193 N). Las de color rojo son las cargas de peso propio de los mismos $(98 ; 67 ; 222 ; 67 ; 222 ; 44$ y $356 \mathrm{~N})$, mientras que las de color azul son la resultante del viento en el Poste y en el Brazo de la estructura (1971 y 2340 N). La de color marrón es el peso propio de toda la estructura (10676,83 N). 
Fig. 5 a) Estructura con Restricciones y Cargas: de viento (eje Z) y peso propio (eje Y) en Newton. b) Detalle de las Restricciones.

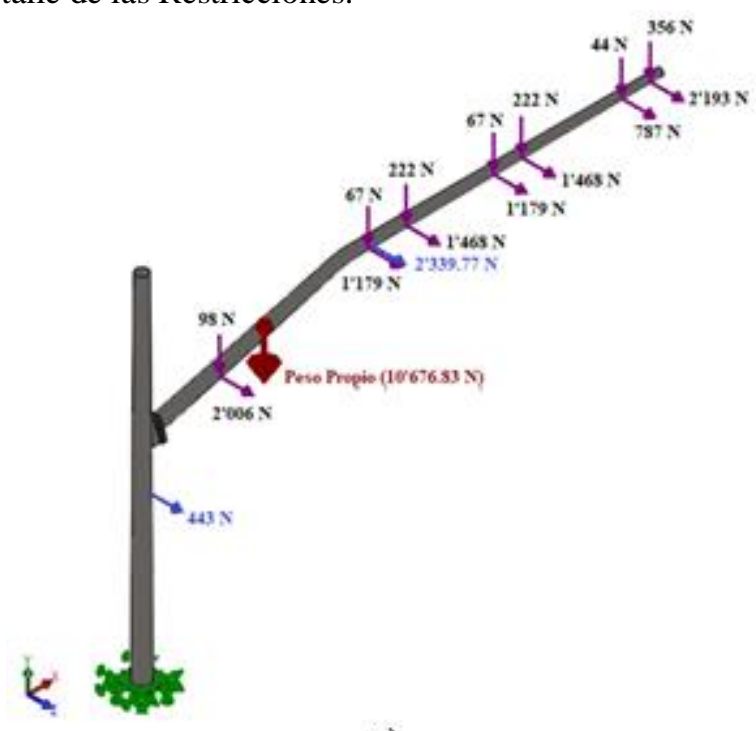

a)

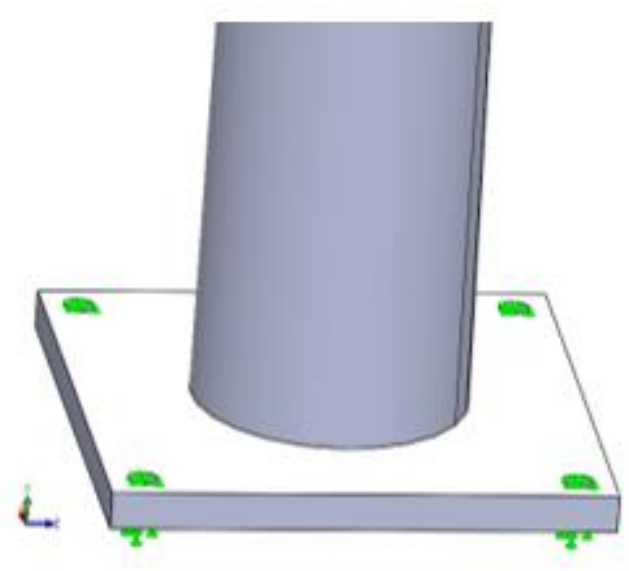

b)

El efecto de estas cargas se manifiesta en el resto de la estructura como fuerzas y momentos. En la figura 6 se muestra el efecto de las cargas de viento y peso propio en el Poste.

Fig. 6 Efectos en el Poste provocados por las cargas de viento $(\mathrm{Pw})$ y el peso propio $(\mathrm{Pp})$.

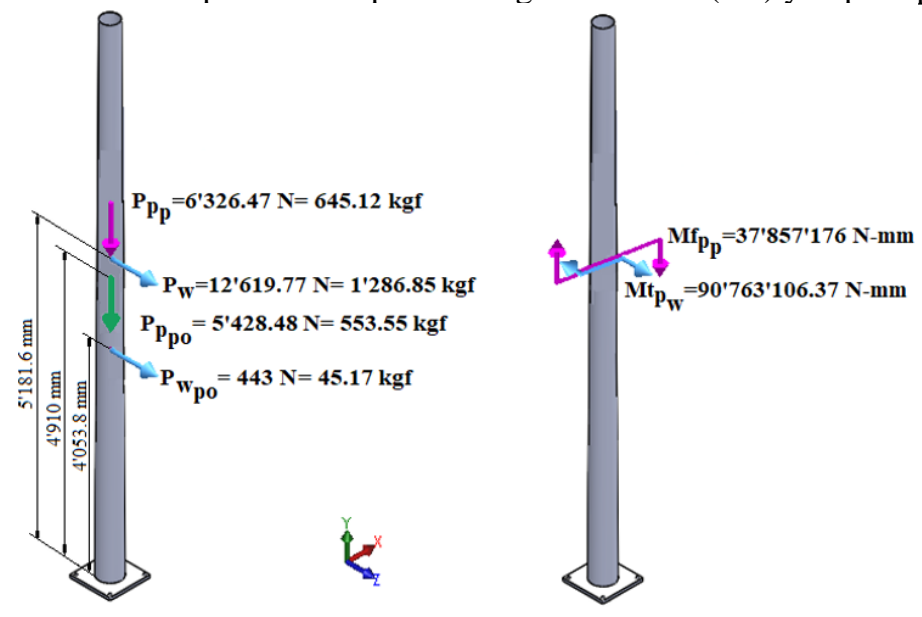

Para poder conocer las fuerzas internas que provocan estas cargas y sus efectos en el Poste se construyeron los gráficos de fuerza normal, fuerza cortante, momento flector y momento torsor. En la figura 7 aparecen estos gráficos. 
Fig. 7 Fuerzas Internas en el Poste.

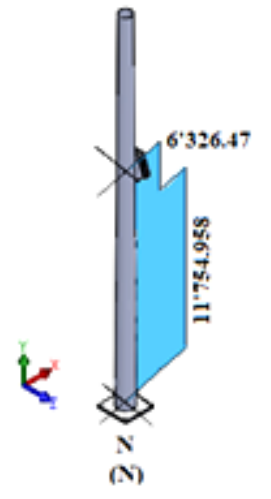

Fuerza Axial

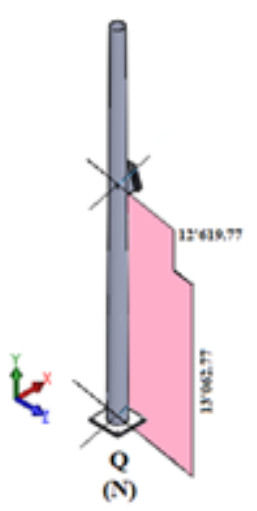

Fuerza Cortante

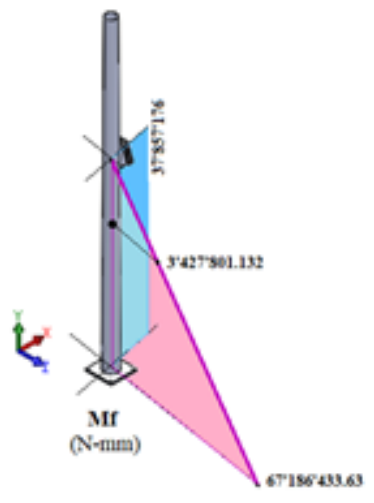

Momento Flector

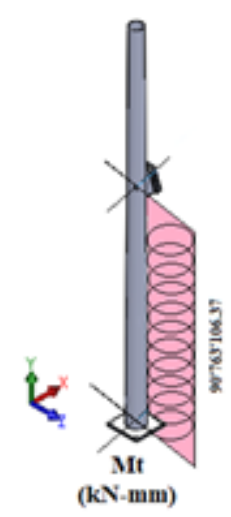

Momento Torsor

A partir de estos momentos se calculan las tensiones equivalentes von Mises para posteriormente compararlas con las tensiones obtenidas mediante la simulación numérica en la base del Poste.

Momento Flector Resultante:

$$
\begin{aligned}
& \mathrm{M}=\sqrt{\mathrm{Mf}_{\mathrm{pp}}{ }^{2}+\mathrm{Mf}_{\mathrm{pw}}{ }^{2}} \\
& \mathrm{M}=\sqrt{37857176^{2}+67186433,63^{2}} \\
& \mathrm{M}=77117978,70 \mathrm{~N}-\mathrm{mm}
\end{aligned}
$$

Módulo de la sección:

$$
\begin{aligned}
& \mathrm{W}_{\mathrm{x}}=\frac{\pi \cdot \mathrm{D}^{3}}{32} *\left(1-\left[\frac{\mathrm{d}}{\mathrm{D}}\right]^{4}\right) \\
& =\frac{\pi \cdot(381)^{3}}{32} *\left(1-\left[\frac{365,125}{381}\right]^{4}\right) \\
& =849512,48 \mathrm{~mm}^{3}
\end{aligned}
$$

Momento Reducido:

$$
\begin{aligned}
& \mathrm{M}_{\text {red-VM }}=\sqrt{0.75 \mathrm{Mt}_{\mathrm{pw}}{ }^{2}+\mathrm{M}^{2}} \\
& \mathrm{M}_{\text {red-VM }}= \\
& \sqrt{\left[0.75(90763106,37)^{2}\right]+\left[(77117978,70)^{2}\right]} \\
& \mathrm{M}_{\text {red-VM }}=110116478,09 \mathrm{~N}-\mathrm{mm}
\end{aligned}
$$

Tensión Equivalente en la base del Poste (según la Teoría de Von Mises):

$$
\begin{aligned}
& \sigma_{\mathrm{EQ}-\mathrm{VM}}=\frac{\text { Mred-VM }}{\mathrm{W}_{\mathrm{X}}}=\frac{110116478.09}{849512,48}= \\
& \sigma_{\mathrm{EQ}-\mathrm{VM}}=129,62 \mathrm{MPa}
\end{aligned}
$$

Valores cercanos a estas tensiones calculadas se pueden apreciar en la figura 8. Los mismos fueron tomados en el nodo 114 de la unión entre el Poste y su base de apoyo (136,7 MPa).

Con el valor del momento torsor y el módulo de la sección calculados anteriormente se determina el valor de la Tensión Tangencial máxima ( $\square$ Máx):

$$
\tau \text { Máx }=\frac{\text { Mt }}{W_{p}}=\frac{90^{\circ} 763^{\prime} 106,37}{2 W_{x}}=\frac{90^{\circ} 763^{\prime} 106,37 \mathrm{~N}-\mathrm{mm}}{2\left(849^{\prime} 512,48\right) \mathrm{mm}}=53.42 \mathrm{MPa}
$$

En la figura 8 se puede apreciar también el valor de tensión tangencial obtenido de la simulación en el nodo 120 de la unión entre el Poste y su Base de apoyo (57,6 MPa). 
Fig. 8 a) Tensión von Mises (nodo 114). b) Tensión Tangencial (nodo 120)

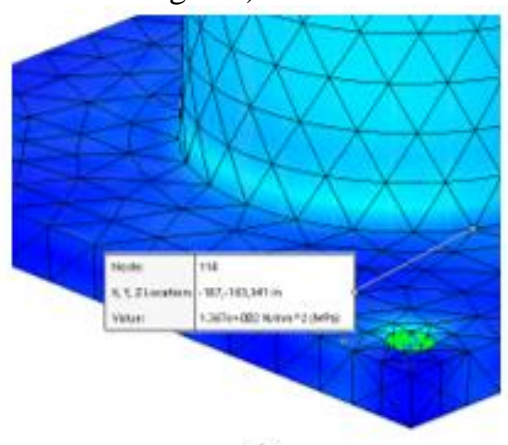

a)

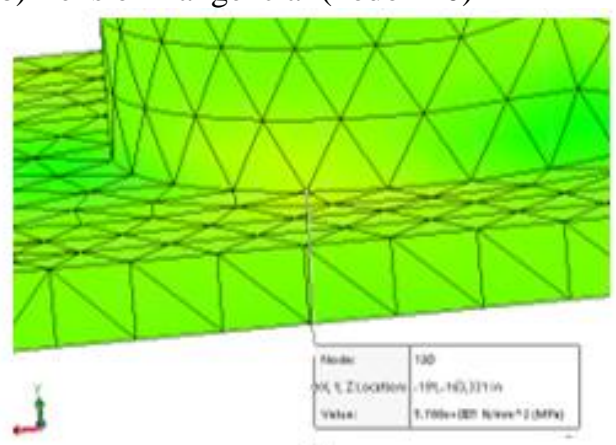

b)

Estos resultados a pesar de ser obtenidos por diferentes vías y métodos son similares, con una diferencia de 7,08 y 4,18 MPa, respectivamente.

\subsubsection{Invariantes: Malla (validación) y Características del Material}

La malla es uno de los elementos más importantes para la simulación por el método de elementos finitos, cada malla que se realice tiene sus propias características. La malla creada para la simulación en la estructura de soporte previamente referida es una malla solida de elementos tetraédricos parabólicos de 10 nodos con un total de 4 puntos Jacobianos. El tamaño de los elementos de dicha malla oscila entre 17 y 54 milímetros (mínimo y máximo) para un total de 48749 elementos y 96234 nodos. En la figura 9 se muestran más detalles. Esta malla de elementos finitos fue validada, es decir, se realizaron 3 mallas: una fina, una media y una gruesa y se ejecutó la simulación para cada una de ellas. Se eligió un lugar geométrico en el cual se obtuvo el valor de la tensión von Mises para cada corrida. La diferencia entre dichos valores de tensión entre dos análisis consecutivos no sobrepasó el 5\%, indicando esto que la precisión es adecuada. Por lo tanto, en este caso particular de análisis, se empleó la malla media que es, además, la propuesta por el software.

Fig. 9 a) Características de la Malla. b) Detalle del modelo geométrico mallado.

\begin{tabular}{|c|c|}
\hline Mesh Details & +4 \\
\hline Study name & Stabic 1 [-Predeteminado-1 \\
\hline Mesthope & Sold Mesh \\
\hline Mesher Used & Blended curvoture-based mesh \\
\hline Jacobian points & 4 points \\
\hline Max Elemenk Size & $54 \mathrm{~mm}$ \\
\hline MnEletrenk Size & $17.0103 \mathrm{~mm}$ \\
\hline Mesh qualy & High \\
\hline Tolal nodes & $\$ 5234$ \\
\hline Tolal eletierks & 48749 \\
\hline Masimum Aspect Ratio & 39989 \\
\hline $\begin{array}{l}\text { Pencentage of elements } \\
\text { whin Aspect Ratio < } 3\end{array}$ & 11.9 \\
\hline $\begin{array}{l}\text { Pexcenlage of elements } \\
\text { why Aspect Ratio> } 10\end{array}$ & 225 \\
\hline $\begin{array}{l}\text { Zof distorted elements } \\
\text { (Jacobian) }\end{array}$ & 0 \\
\hline Remesh laled patts with incompatible mesh & OH \\
\hline Time to complete mest([hummuss] & $0000-14$ \\
\hline
\end{tabular}

a)

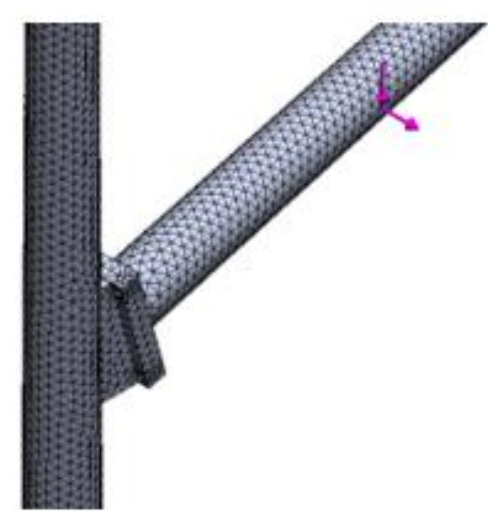

b) 
Toda la estructura está constituida de tres tipos de acero: los tubos de acuerdo a la norma ASTM A500 Grade B [20], las placas de unión entre el Brazo y el Poste según la norma ASTM A36 [21] y la placa base de ASME STS-1 [22-24]. Las características más importantes e imprescindibles para la simulación de los dos primeros materiales aparecen en la tabla 1.

Tabla 1: Características más importantes de los materiales de los tubos y las placas. Fuente: [20] [21] [22]

\begin{tabular}{l|c|c} 
& $\begin{array}{c}\text { Tubos } \\
\text { (ASTM A500, Grade B) }\end{array}$ & $\begin{array}{c}\text { Placas de unión y Placa base } \\
\text { ASTM A36 Steel, flange }\end{array}$ \\
\hline Módulo de Elasticidad $-E_{s}(\mathrm{ksi})$ & $29^{\prime} 000$ & $29^{\prime} 000$ \\
Límite de Fluencia $-F_{y}(\mathrm{ksi})$ & 48 & 36 \\
Peso específico $-\square_{\text {steel }}\left(\mathrm{lbf} / \mathrm{ft}^{3}\right)$ & 490 & 480
\end{tabular}

\section{RESULTADOS E DISCUSSÃO}

\subsection{TENSIONES Y DESPLAZAMIENTOS}

El comportamiento de las tensiones von Mises de toda la estructura puede ser apreciado en la figura 10. El valor máximo de estas tensiones es de $1211 \mathrm{MPa}$ y se encuentra en un punto situado en el interior del Poste en la zona de la unión Poste-Brazo. Esa intersección geométrica compleja entre ambos tubos, unidos por dos placas de $50,8 \mathrm{~mm}$ cada una, hacen que el comportamiento de esa zona funcione como un empotramiento que soporta las cargas que actúan en el Brazo y que llegan hasta dicho punto. En esa zona, como se pudo apreciar previamente aparecen fuerzas normales, fuerzas cortantes, momentos flectores y torsores, siendo estos los que motivan que aparezcan puntos con esos valores de tensiones que sobrepasan el límite del material (330,9 MPa).

De esta misma manera ocurre con las tensiones tangenciales $\square_{\mathrm{xz}}$ que alcanzan un valor máximo de 324,5 MPa que también sobrepasa la tensión admisible a cortante del material del poste (198,57 MPa). En el resto de la estructura el comportamiento tensional es adecuado.

Como se muestra en la figura 11 el mayor valor de desplazamiento se encuentra en la zona más alejada de la Base del Brazo, es decir, en la punta del Brazo con 1329 mm. En estos desplazamientos tienen una mayor influencia las cargas de viento que actúan sobre la estructura. Se debe tener en cuenta que esta simulación se realizó con valores de carga de vientos extremos, es decir, vientos con velocidades de $185 \mathrm{~km} / \mathrm{h}$ y por otro lado la estructura posee un voladizo de $12,19 \mathrm{~m}$, lo que justifica estos resultados del desplazamiento. 
Fig. 10 a) Tensiones equivalente Von Mises (MPa). b) Tensiones tangenciales máximas $\square_{\mathrm{xz}}(\mathrm{MPa})$.

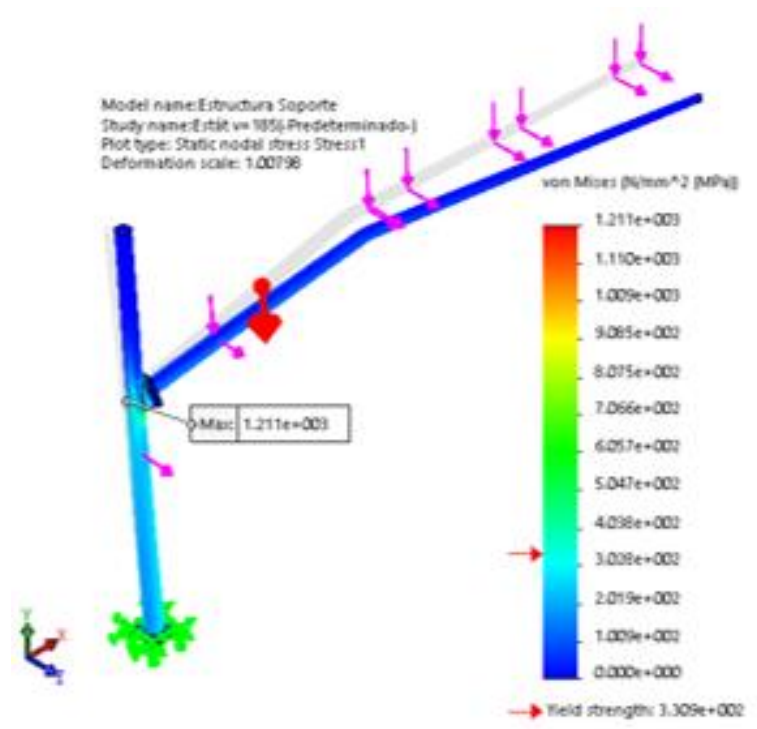

a)

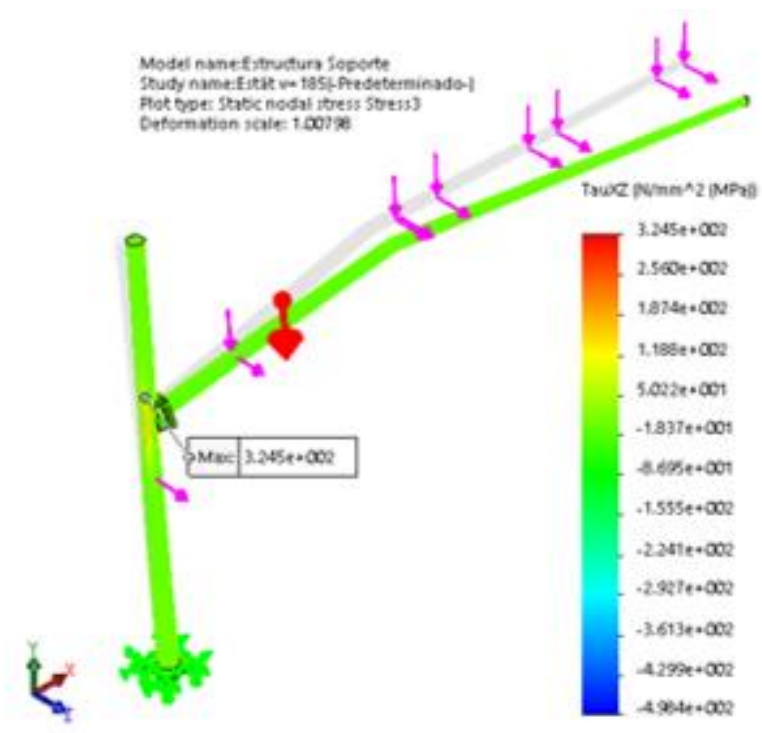

b)

Fig. 11 Desplazamientos resultantes (mm).

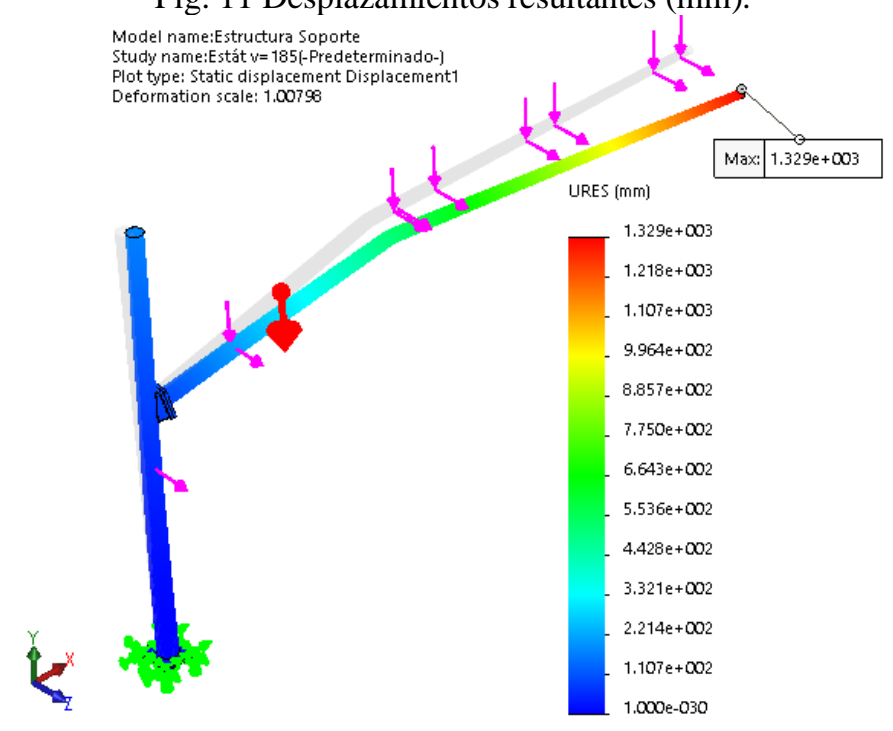

\subsection{ESTABILIDAD}

Teniendo en cuenta que el Poste está sometido a una carga de compresión proveniente de los efectos de los pesos propios de la estructura, de los semáforos y señales y que la sección transversal del mismo es anular con diámetro exterior de $381 \mathrm{~mm}$ y espesor de 7,9375 mm se decidió hacer la comprobación de la estabilidad de este elemento [25]. Algunos resultados de este estudio se pueden observar en la figura 12. 
Fig. 12 Algunos resultados del estudio de Estabilidad

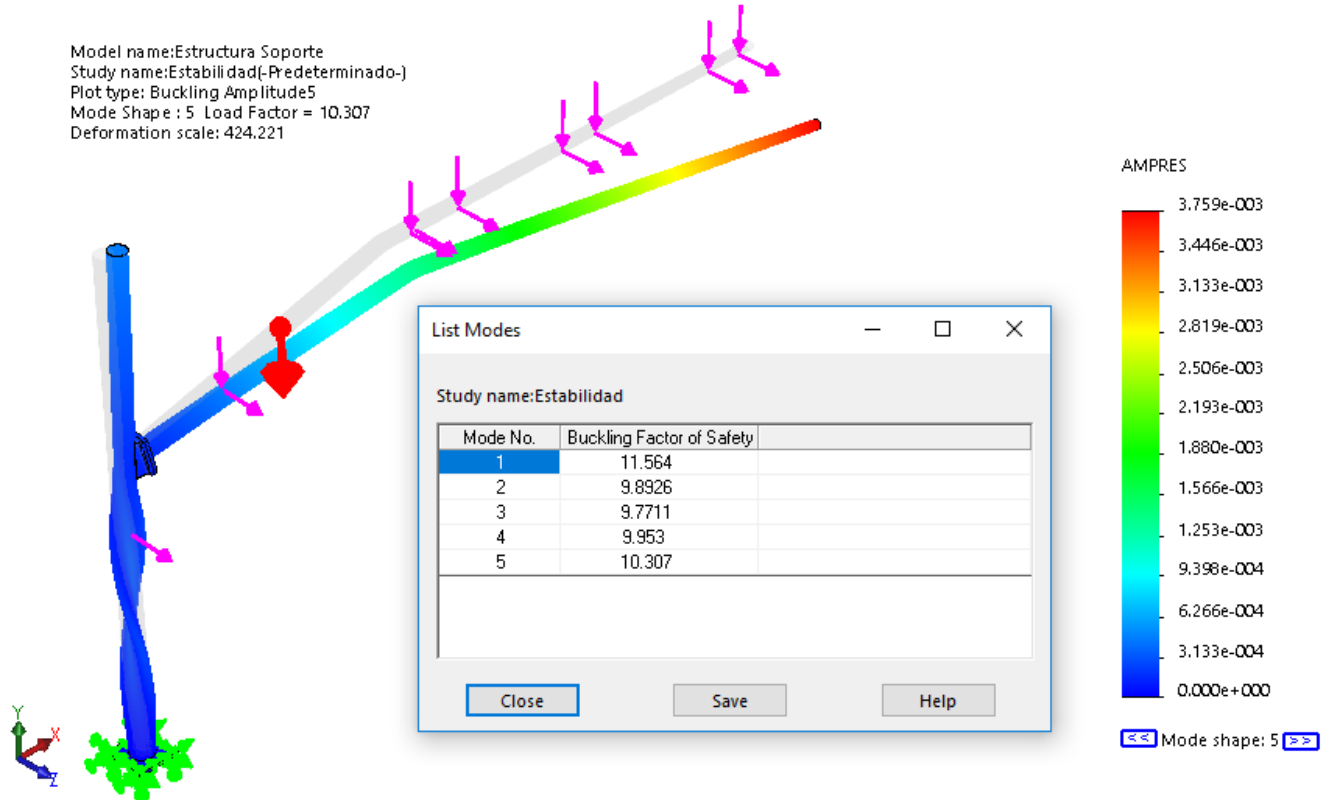

De todo este estudio se muestra solamente la Amplitud Resultante (AMPRES) del 5to Modo y el Factor de Seguridad a Estabilidad (Buckling Factor of Safety) para cada Modo, el cual oscila alrededor 10, por lo que se puede afirmar que el Poste no tendrá problemas de estabilidad.

\subsection{ANÁLISIS MODAL}

Como se mencionó anteriormente, en la ciudad donde se encuentra la estructura calculada pueden ocurrir vientos de $185 \mathrm{~km} / \mathrm{h}$, los cuales representan vientos huracanados de categoría 3 de acuerdo a la escala Saffir-Simpson. Bajo esta categoría los daños se consideran extensos. Por todo ello, se decide hacer el análisis modal de la estructura para comprobar la ocurrencia o no del fenómeno de resonancia.

Se calculan las frecuencias propias o naturales, cuyos valores en Hertz son: 0,92965; 1,1266; 3,2906; 3,6497 y 8,7762. El software empleado al calcular las frecuencias tiene en cuenta el peso propio de la estructura, es decir, no es necesario especificárselo como ocurre en otros softwares. En la figura 13 se puede observar el primer modo de oscilación y un gráfico de Frecuencia (Hz) vs. Número de Modo. 
Fig. 13 a) Primer modo de oscilación. b) Gráfico de Frecuencia (Hz) vs. Número de Modo

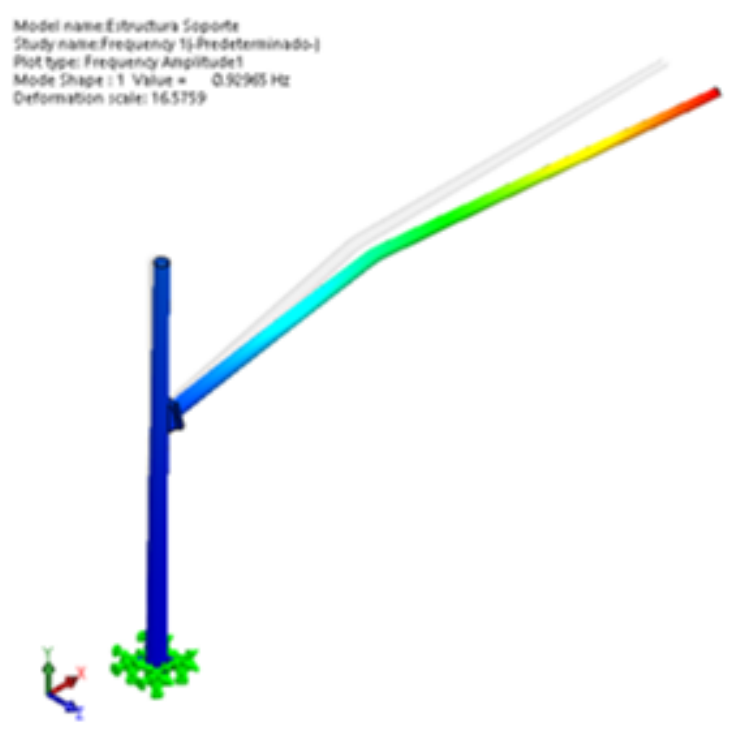

a)

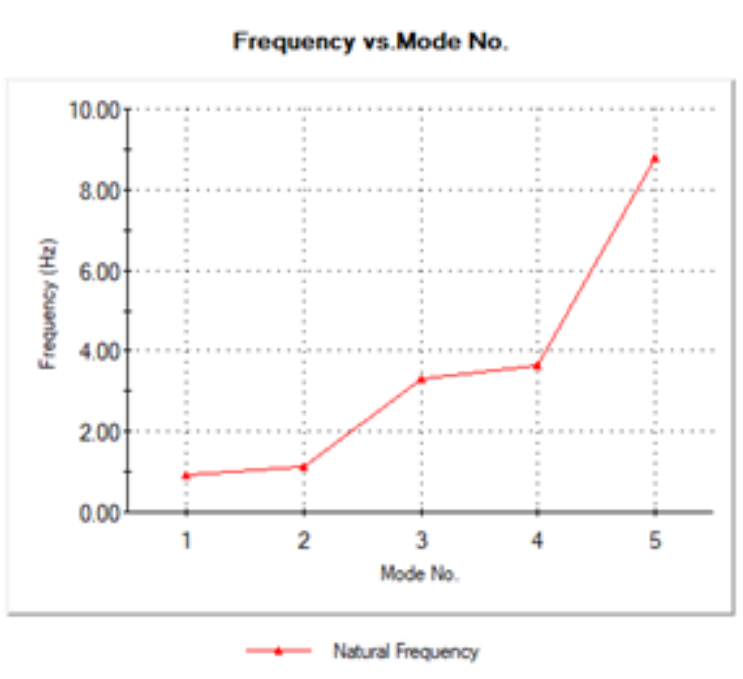

b)

Posteriormente se calcularon las frecuencias y los modos bajo los efectos del sistema de cargas descrito anteriormente y mostrado en la figura 5. Al comparar los valores de las frecuencias obtenidos por las dos vías no hay coincidencia en ninguno de los modos, por lo que se puede afirmar que el fenómeno de resonancia no ocurrirá en esta estructura.

También se obtuvieron las curvas de variación de los desplazamientos y las tensiones (von Mises y tangenciales) de acuerdo a la velocidad del viento (ver Fig. 14), es decir, se fue variando la velocidad del viento de acuerdo a los diferentes valores de la escala Saffir-Simpson (118-153, 154177, 178-209, 210-250 y >250 km/h) y haciendo el análisis modal. Para el rango de la categoría 3 (178209) se tomó $185 \mathrm{~km} / \mathrm{h}$ que es el valor de la velocidad del viento con que se calcula la estructura evaluada. Para ello fue necesario calcular el valor de las componentes de las fuerzas (Pw_poste, Pw_brazo, Pw_semáforos y Pw_señales) para cada velocidad del viento escogida y realizar las cinco simulaciones. 
Fig. 14 Variación de los desplazamientos y las tensiones según la velocidad del viento.

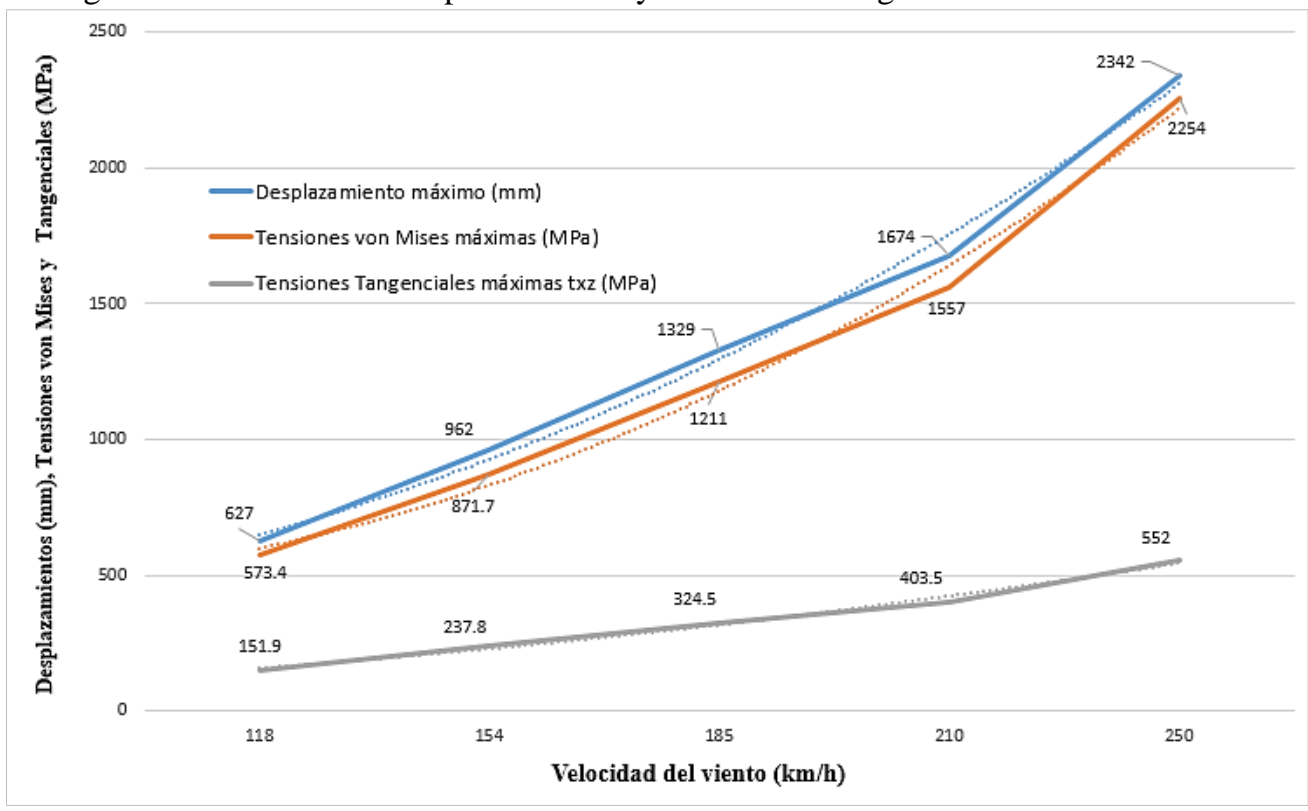

En las curvas de la figura anterior se aprecia que las tres variables (desplazamientos, tensiones von Mises y tensiones tangenciales) aumentan con cierta proporcionalidad en la medida que aumenta la velocidad del viento. En la zona cercana a $210 \mathrm{~km} / \mathrm{h}$ hay un cambio de pendiente y el aumento es más brusco. Las ecuaciones polinómicas de orden 2 que describen el comportamiento de dichas variables son las siguientes:

$$
\begin{array}{ll}
\text { Dessplazamiento máximo }(\mathrm{mm}): & \boldsymbol{\delta}=46 x^{2}+138,2 x+466,2 \\
\text { Tensión equivalente von Mises máxima (MPa): } & \boldsymbol{\sigma}_{\mathrm{EQ}-\mathrm{VM}}=57,436 x^{2}+60,036 x+481,52 \\
\text { Tensión tangencial máxima (MPa): } & \boldsymbol{\tau}_{\mathrm{za}}=8,3929 x^{2}+46,233 x+102,92
\end{array}
$$

En cada ecuación la variable $\boldsymbol{x}$ es la velocidad del viento. Todas estas ecuaciones se obtuvieron con un $\mathrm{R}^{2}$ muy cercano a la unidad, por lo que la precisión de las mismas está asegurada.

\section{CONCLUSÕES}

La metodología de cálculo analítico mediante la norma AASHTO para las estructuras de soporte de las señalizaciones viales fue automatizada en hojas de cálculo Excel y comprobada con una estructura real. La simulación numérica efectuada en este trabajo permitió conocer diversos parámetros como son las tensiones von Mises y el desplazamiento, entre otros, que la metodología de cálculo analítico mediante la norma AASHTO no lo permite.

Las tensiones von Mises obtenidas son elevadas en algunos puntos debido a la compleja configuración geométrica de la unión Brazo-Poste. Sin embargo, el resto de la estructura presenta un 
adecuado comportamiento tensional. Los desplazamientos también son considerables debido a que la simulación se efectuó con elevadas cargas de viento, similares a las que ocurren en el lugar donde se encuentra la estructura ejemplo del Reporte utilizado.

El resultado del cálculo de estabilidad del Poste fue satisfactorio. Mientras que el cálculo de las frecuencias naturales de oscilación bajo cargas, mostró que no ocurre el fenómeno de resonancia.

Fueron obtenidas las curvas de variación de los desplazamientos y las tensiones (von Mises y tangenciales) según la velocidad del viento, así como las ecuaciones que describen su comportamiento.

Todos los resultados mencionados anteriormente, obtenidos a partir de la simulación numérica del modelo de elementos finitos creado, complementan los resultados que se obtienen por las normas. 


\section{REFERÊNCIAS}

[1] Recuenco, P. Historia de las señales de tráfico: Origen y primeros pasos de las señales de tráfico. 2015. Autofácil. Editorial LUIKE Iberoamericana de Revistas, SLU, Madrid, España.

[2] American Association of State Highway and Transportation Officials (AASHTO). LRFD Specifications for Structural Supports for Highway Signs, Luminaires, and Traffic Signals (1st Edition), with 2017, 2018, 2019 and 2020 Interim Revisions. USA: AASHTO; ISBN: 978-1-68015-825-0. 2019.

[3] Garlich MJ, Thorkildsen ET, Guidelines for the installation, inspection, maintenance and repair of structural supports for highway signs, luminaires and traffic signals. Chicago, Illinois, USA: Office of Bridge Technology Federal Highway Administration; Technical Report FHWA NHI 05-036. 2005.

[4] Queiroz R. D., Lima R. G., Garcia del Pino G., Mestriner L. A., Takata E. T; Análise do desgaste do polietileno do componente acetabular da prótese total do quadril, utilizando o método de elementos finitos de simulação computadorizada; Revista Brasileira de Ortopedia. 2001;36(5): 149-154.

[5] Silva A. R. V., Macêdo N.J. C., Miranda A. G., Verçosa L. A., Garcia del Pino G., Rodrigues R. A., Nascimento D. A., Influência do tratamento térmico de normalização na microestrutura e propriedades mecânicas do aço SAE 1035 utilizado em motocicletas, ATENAS. 2021. DOI: 10.22533/at.ed.6222105043.

[6] Filizzola D. M., Santos T. S., Miranda A. G., Costa J. C. M., Nascimento N. R, Santos M. D., Bello R.H., Garcia del Pino G., Macedo N. J. C. Annealing Effect on the Microstructure and Mechanical Properties of AA 5182 Aluminum Alloy. Materials Research. 2021;24(4) DOI: 10.1590/1980-5373-MR2020-05.

[7] Mendes C. S., Garcia del Pino G., Bezazi A., Boumediri H., Neto J. C., Torres A. R., Kieling A. C., Garcia S. D. Comparative performance comparison of Toolox44 tool steel versus SAE H13 for hot forging. Latin American Journal of Development. 2021;3(3):1181-1197. DOI: 10.46814/lajdv3n3-014.

[8] Garcia del Pino G., Bezazi A., Boumediri H., Kieling A. C., Silva C. C., Dehaini J., Rivera J. L. V., Valenzuela M. G. S., Diaz F. R. V., Panzera T, H., Hybrid epoxy composites made from treated curauá fibres and organophilic clay. Journal of Composite Materials. 2020;1(0):1-13. DOI: $10.1177 / 0021998320945785$.

[9] Garcia del Pino G., Kieling A. C., Bezazi A., Panzera T. et al. Hybrid polyester composites reinforced with curauá fibres and nanoclays, Fibres and Polymers. 2020;21(2):399-406. DOI 10.1007/s12221-0209506-7

[10] Constantinescu G, Bhatti A, Phares B, Effect of Wind Induced Unsteady Vortex Shedding, Diurnal Temperature Changes, and Transit Conditions On Truss Structures Supporting Large Highway Signs. Iowa, USA: Iowa Department of Transportation and Federal Highway Administration; 2018. Final Report TR687.

[11] Arabi S, Shafei B, Phares B, Fatigue analysis of sign-support structures during transportation under road-induced excitations. Engineering Structures. 2018;164:305-315. ISSN 0141-0296.

DOI 10.1016/j.engstruct.2018.02.031.

[12] Seo J, Amatya I, Fatigue load testing of full-scale dynamic message signs. Engineering Structures. 2021; 226:415-425. ISSN 0141-0296. DOI 10.1016/j.engstruct.2020.111357.

[13] Puckett JA, Garlich MG, Nowak A, Development and calibration of AASHTO LRFD Specifications for structural supports for highway signs, luminaires and traffic signals. Washington, D.C., USA: American 
Association of State Highway and Transportation Officials; 2014. NCHRP Report 796. ISBN 978-0-30930818-2

[14] Fouad H, Davidson JS, Delatte N, Structural supports for highway signs, luminaires, and traffic signals. Washington, D.C., USA: American Association of State Highway and Transportation Officials; 2003. NCHRP Report 494. ISBN 0-309-08753-8

[15] Dexter, RJ, Ricker, HJ, Fatigue Resistant Design of Cantilevered Signal, Sign and Light Supports, Transportation Research Board. Washington, D.C., USA: American Association of State Highway and Transportation Officials; 2002. NCHRP Report 469. ISBN 0-309-06724-3

[16] Chung C. Fu, Yunchao Ye, Chaoran Xu, The Calibration of the AASHTO ASD and LRFD for Maryland Sign and High Mast Lighting Structure Design. Maryland, USA: Federal Highway Administration or the Maryland Department of Transportation; 2020. MD-20-SHA/UM/5-01 Tech Report.

[17] Chagoyen- C. A M.; Acosta- Y G.; Rodríguez Y. T, Recarey-M., C., Aragón G. B L. \& Leiva J. P.; Simulación numérica de los apoyos de puente metálico giratorio. Ingeniería Mecánica, 2011;14(3):170180. (2011).

[18] Arabi S, Shafei B, Multi-stressor fatigue assessment of steel sign-support structures: A case study in Iowa, Engineering Structures, 2019;200:610-620. ISSN 0141-0296. DOI 10.1016/j.engstruct. 2019.109721.

[19] Arabi S, Shafei B, Phares B, Investigation of fatigue in steel sign-support structures under diurnal temperature changes, Journal of Constructional Steel Research, 219;153:286-297. ISSN 0143-974X. DOI 10.1016/j.jcsr.2018.09.024.

[20] ASTM A500 Grade B. Standard Specification for Cold-Formed Welded and Seamless Carbon Steel Structural Tubing in Rounds and Shapes, American Society for Testing Materials International, West Conshohocken, PA, United States. 2021.

[21] ASTM A36, flange. Standard Specification for Carbon Structural Steel, American Society for Testing Materials, West Conshohocken, PA, United States, 2019.

[22] ASME STS-1-06 Standard, Steel Stacks. The American Society of Mechanical Engineers, N.Y., 108 p. ASME Steel Stacks Committee. (2016).

[23] Machado Rodríguez, Ángel Silvio, Kido Shimomoto, Eric, Silverio, Rubens, Garcia del Pino, Gilberto, Chagoyen Méndez, César Alberto, \& Moya Rodríguez, Jorge Laureano. (2017). Sinterización, un proceso de conformaci $\tilde{A}^{3} \mathrm{n}$ de metales como alternativa económica con un bajo impacto ambiental. Centro Azúcar, 2017;44(3):83-92.

[24] Soares C. G. C.; Garcia del Pino G.; Bezazi A.; Estudo comparativo entre transmissões automotivas automáticas e manuais; Latin American Journal of Development, v. 3, n. 4, p. 2801-2818, v. 3, n. 5; 2021.

[25] Mawa Dalia Z, Bhowmick AK, Grondin GY, Local buckling of multi-sided steel tube sections under axial compression and bending, Journal of Constructional Steel Research, 2021;186:180-190. ISSN 0143974X. DOI 10.1016/j.jcsr.2021.106909. 